\title{
Multiple zoonotic helminth infections in domestic dogs in a rural area of Khuzestan Province in Iran
}

\author{
Molouk Beiromvand ${ }^{1,2^{*}}$ (D, Abdollah Rafiei ${ }^{1,2}$, Elham Razmjou ${ }^{3}$ and Sharif Maraghi ${ }^{1,4}$
}

\begin{abstract}
Background: Echinococcosis and toxocarosis caused by the genus of Echinococcus and Toxocara spp. are among important helminthic diseases worldwide. Limited data on the prevalence of these parasites persuaded us to determine the prevalence of E. granulosus, E. multilocularis, and T. canis infections in domestic dogs in rural areas of Ahvaz, southwestern Iran. Fecal samples from 167 domestic dogs were examined using both microscopy and PCR techniques. Multiplex PCR was performed for the presence of Echinococcus, and Taenia spp. and single PCR for detection of $T$. canis and Toxascaris leonina.

Results: The total occurrence of identified parasites was 65 (38.9\%). The microscopic examinations showed that 40 (24\%), 18 (10.8\%), and four (2.4\%) of dogs were infected with taeniid-like, ascarid, and both genera eggs, respectively. Echinococcus granulosus was identified in seven (4.2\%), Taenia spp. in 29 (17.4\%), and mixed infection with both in 11 (6.6\%) samples. Sequencing of PCR-positive samples identified E. granulosus s.s. (G1), 18 T. hydatigena (10.8\%), five T. multiceps (3\%), three T. serialis (1.8\%), one T. ovis (0.6\%), one Spirometra erinaceieuropaei voucher $(0.6 \%)$, and two Mesocestoides corti (1.2\%). This is the first report of S. erinaceieuropaei voucher and M. corti in dogs in Iran. Nine (5.4\%) and 16 (9.6\%) dogs showed infection with T. canis and T. leonina, respectively. Two samples showed coinfection with both ascarids.

Conclusions: Several studies have reported echinococcosis and toxocarosis in intermediate hosts from the southwest of Iran; however, this study is the first molecular research on E. granulosus and T. canis in domestic dogs in a rural area of southwestern Iran. Furthermore, issues of soil contamination with dogs' feces and recent dust storms in Khuzestan may have a role in the spreading of these zoonotic infections to other provinces close to it, and neighboring countries such as Iraq.
\end{abstract}

Keywords: Echinococcus spp., Taenia spp., Toxocara canis, Toxascaris leonina, Spirometra erinaceieuropaei voucher, Mesocestoides corti., Dog, Iran

\section{Background}

The domestic dog as final host of zoonotic enteric parasites plays an important role in the transmission of diseases such as echinococcosis and toxocarosis to humans [1]. Infected dogs can contaminate the environment with excreted eggs, posing a serious public health problem [2]. Toxocarosis, an important helminthic disease, is caused by the larval stages

\footnotetext{
* Correspondence: Beiromvand-m@ajums.ac.ir

${ }^{1}$ Infectious and Tropical Diseases Research Center, Health Research Institute,

Ahvaz Jundishapur University of Medical Sciences, Ahvaz, Iran

${ }^{2}$ Department of Parasitology, School of Medicine, Ahvaz Jundishapur

University of Medical Sciences, Ahvaz, Iran

Full list of author information is available at the end of the article
}

of the nematodes Toxocara canis and Toxocara cati, the adult forms of which reside in the small intestine of canine and feline hosts, respectively [3]. The worldwide occurrence of $T$. canis in dogs is reported to range from 3.1 to $82.6 \%$ [4-7]. Humans can acquire the infection through the ingestion of embryonated eggs via geophagia, contaminated food, and raw vegetables or by ingestion of undercooked meat of infected paratenic hosts $[1,8]$. Echinococcosis, an important zoonotic disease with worldwide distribution, is caused by the metacestode stage of tapeworms belonging to Echinococcus spp. [9]. Recently, the disease has been included in a subgroup of 17 neglected tropical diseases by the World Health Organization [10]. Cystic

(c) The Author(s). 2018 Open Access This article is distributed under the terms of the Creative Commons Attribution 4.0 International License (http://creativecommons.org/licenses/by/4.0/), which permits unrestricted use, distribution, and 
echinococcosis (CE) and alveolar echinococcosis (AE), caused by E. granulosus and E. multilocularis, respectively, have particular medical, economic, veterinary, and public health importance [9].

The life cycle of these cestodes involves dogs and other canids as definitive hosts with domestic and wild ungulates as intermediate hosts of E. granulosus and small mammals as intermediate host of $E$. multilocularis. Humans can act as an accidental intermediate hosts through ingestion of Echinococcus eggs, which are excreted into the environment in feces of final hosts. Based on molecular studies using mitochondrial genes, 10 strains (genotypes G1-G10) have been recognized, including E. granulosus sensu stricto (s.s.) (genotypes G1-G3), Echinococcus equinus (the 'horse strain', genotype G4), Echinococcus ortleppi (the 'cattle strain', genotype G5), and Echinococcus canadensis (G6-G10) [11, 12]. The genotype G1 is the most common genotype on all continents and the primary strain infecting humans [13]. More than 270 million people living in the Central Asian Republics, Afghanistan, Pakistan, China, and parts of Iran, particularly rural inhabitants, principally farmers and herdsmen, are at risk of CE [14]. Cystic echinococcosis is also one of the most frequently reported zoonoses and an important public health problem in the Middle East $[15,16]$.

Iran is an endemic focus of $\mathrm{CE}$ and $\mathrm{AE}[16,17]$. Hospital records indicate that approximately $1 \%$ of patients seen in surgical wards in Iran are infected with E. granulosus [16]. The incidence of human $C E$ in Iran is estimated to be 1.18-3 per 100,000 population [18]. An E. granulosus infection prevalence of $5-49 \%$ in stray dogs, $88 \%$ in sheep, $70 \%$ in camels, and 19\% in cattle has been recorded in Iran [16]. Echinococcus granulosus s.s. (G1-G3) and the camel strain (G6) are causative agents of canine and human echinococcosis in Iran [13, 19], and, in some parts of the country, the G1 strain is the cause of $75-100 \%$ of canine infections [20]. Echinococcus multilocularis infection was first reported in 1970 in the northwest of Iran [21, 22], and the latest survey in northeastern country revealed $E$. multilocularis infection in domestic and wild canids [17]. Although southwestern Iran is not an endemic area of $\mathrm{AE}$, a human case of $\mathrm{AE}$ was reported for the first time in 2012 [23].

In many rural areas of Iran, large populations of dogs are kept for being shepherd dogs and guard dogs, and the prevalence of E. granulosus infection in up to $64 \%$ in dogs play key roles in the transmission of echinococcosis to humans [14, 24]. A purgation study using arecoline hydrobromide in 13 provinces indicated that 20\% of farm dogs in Khuzestan Province were infected with $E$. granulosus [24]. In Khuzestan villages, increasing the dog population, which roam gardens and streets and the lack of hygienic disposal of dog's feces from the ground lead to soil contamination with intestinal parasites [24, 25]. Moreover, the province had been hit by dust storms many times in recent years [26], which may increase the risk of human infections to soil transmitted pathogens in the rural inhabitants. In order to control the parasitic diseases in this region, new data on the existence of parasitic agents, their prevalence, and transmission routes are considered necessary. Thus, this study was performed using the flotation/sieve technique, microscopy and PCR to determine the prevalence of E. granulosus, E. multilocularis, and T. canis and Toxascaris leonina infections in order to identify the role of domestic dogs in the environmental contamination and transmission of the infections in rural areas of Ahvaz, southwestern Iran.

\section{Methods}

\section{Study area}

Khuzestan Province, one of the 31 provinces of Iran, located in the southwest area of the country near the borders with Iraq and the Persian Gulf, has an area of $63,238 \mathrm{~km}^{2}$ and a population of $4,531,720$ [27]. The landscape comprises rolling hills and mountainous regions in the north with plains and marshlands to the south. The capital, Ahvaz, $\left(31^{\circ} 19^{\prime} \mathrm{N} 48^{\circ} 40^{\prime} \mathrm{E}\right)$ has a population of 1,112,021 [27]. Three of the country's largest rivers, the Karoun, Karkheh, and Maroun, make the Khuzestan plain especially suited for agriculture. The climate is hot and occasionally humid. The annual mean of maximum and minimum temperatures is about $50{ }^{\circ} \mathrm{C}$ and $9{ }^{\circ} \mathrm{C}$ in months of July and March, respectively. The annual precipitation is $150-256 \mathrm{~mm}$ in the south and 995-1100 $\mathrm{mm}$ in the north [26].

\section{Sampling}

The feces of 167 domestic dogs were collected from rural areas of Ahvaz County, the capital of Khuzestan Province in 2013 and 2014. Simple random sampling was carried out according to information from the local veterinary offices. All samples were placed in zippered plastic bags, labeled with an assigned number, location, and date of sampling and immediately taken to the Parasitology Department, Ahvaz Jundishapur University of Medical Sciences, Iran. For safety and to kill oncospheres of Echinococcus spp. eggs [28], all samples were frozen at $-80{ }^{\circ} \mathrm{C}$ for at least 7 days, and then held at $-20{ }^{\circ} \mathrm{C}$ until examination.

\section{Egg isolation by flotation/sieving}

Taeniid-like and ascarid eggs were isolated from feces by flotation with a zinc chloride solution (density, $1.45 \mathrm{~g} / \mathrm{ml}^{-1}$ ). Approximately $5 \mathrm{~g}$ of fecal sample were suspended in $50 \mathrm{ml}$ tap water and mixed until completely dispersed. The fecal suspensions were filtered through $100 \mu \mathrm{m}$ mesh to remove large particles, and, subsequently centrifuged at $1000 \mathrm{~g}$ for $5 \mathrm{~min}$. After discarding the supernatant $12 \mathrm{ml}$ saturated zinc chloride solution was added to fecal sediment and carefully 
vortexed. Afterwards, the suspension was centrifuged at $1000 \mathrm{~g}$ for $30 \mathrm{~min}$, and then sequential sieving through 40 and $21 \mu \mathrm{m}$ mesh was used for isolating ascarid and taeniid eggs, respectively as described by Mathis et al. [29]. Identification of taeniid and ascarid eggs was performed by light microscopy and then the rest of the sediments (approximately $200 \mu \mathrm{l}$ ) were transferred to $2 \mathrm{ml}$ tubes and stored at $-20{ }^{\circ} \mathrm{C}$ until examination.

\section{DNA extraction}

DNA isolation of all samples was performed using the QIAamp DNA Mini Kit (QIAGEN, Germany) according to the manufacturer's instructions with slight modification of the final step for both taeniid-like and ascarid eggs $[17,30]$ and increase of incubation time at $56{ }^{\circ} \mathrm{C}$ to at least $16 \mathrm{~h}$ for ascarid eggs until eggs were completely lysed [31]. Before extraction, five cycles of freezing in liquid nitrogen $5 \mathrm{~min}$ and boiling at $100{ }^{\circ} \mathrm{C} 7 \mathrm{~min}$ were conducted to disrupt the parasite egg wall. Duration of the final step was increased to $5 \mathrm{~min}$ to improve the yield of DNA [17]. DNA extracts were stored at $-20{ }^{\circ} \mathrm{C}$ until PCR was conducted.

\section{PCR}

Multiplex PCR was conducted on all samples for the amplification of the $395 \mathrm{bp}$ fragment of NADH dehydrogenase subunit 1 (nad1) gene, in order to detect E. multilocularis using the primer pairs Cest1 (5'-TGCTGATTTGTTAAAGTTA GTGATC-3') and Cest2 (5' -CATAAATCAATGGAAACAA CAACAAG-3'), and 117 bp of the small subunit of ribosomal RNA (rrnS) gene to identify E. granulosus using the primers Cest4 (5'-GTTTTTGTGTGTTACATTAATAAG GGTG-3') and Cest5 (5'-GCGGTGTGTACMTGAG CTAAAC-3'), and also Taenia spp. to identify 267 bp using the primer sets Cest3 (5'-YGAYTCTTTTTAGGGGAA GGTGTG-3') and Cest5 (5'-GCGGTGTGTACMTGAG CTAAAC-3') in conditions as described previously [32]. The PCR reaction was performed in a $25 \mu \mathrm{l}$ volume reaction using $12.5 \mu \mathrm{l}$ of the master mix (QIAGEN Multiplex PCR, Germany), $8 \mu \mathrm{l} \mathrm{H} \mathrm{H}_{2} \mathrm{O}, 2 \mu \mathrm{l}$ DNA template, and $2.5 \mu \mathrm{l}$ of primers ( $2 \mu \mathrm{M}$ of primers Cest 1 , Cest2, Cest3, and Cest 4 and $16 \mu \mathrm{M}$ of primer Cest5 in $\mathrm{H}_{2} \mathrm{O}$ ) [32].

PCR amplification was performed targeting the internal transcribed spacer 2 (ITS-2) regions of the ribosomal DNA genes to amplify 380 and 300 bp fragments for the identification of $T$. canis and T. leonina, using the forward primers Tcan 1 (5'-AGTATGATGGGCGC GCCAAT-3') and Tleo1 (5'-CGAACGCTCATATA ACGGCATACTC-3'), respectively and reverse primer NC2 (5' 'TTAGTTTCTTTTCCTCCGCT-3') on all fecal samples [33]. Amplifications were carried out on a $25 \mu \mathrm{l}$ reaction volume containing $12.5 \mu \mathrm{l}$ of the master mix (Qiagen, Hilden, Germany), $9 \mu$ l distilled water, $2.5 \mu \mathrm{l}$ DNA template, and $0.5 \mu \mathrm{l}$ of each primer under the following described protocol with slight modifications: An initial denaturation step at $95{ }^{\circ} \mathrm{C}$ for $5 \mathrm{~min}$; 35 cycles of $94{ }^{\circ} \mathrm{C}$ for $20 \mathrm{~s}, 58{ }^{\circ} \mathrm{C}$ for $30 \mathrm{~s}$, and $72{ }^{\circ} \mathrm{C}$ for $30 \mathrm{~s}$; and a final extension cycle at $72{ }^{\circ} \mathrm{C}$ for $10 \mathrm{~min}$ [33]. For each amplification, one negative control (distilled water) as well as one positive control contains DNA of E. multilocularis, E. granulosus, Taenia hydatigena, T. canis, and T. leonina sample was included.

PCR amplicons of 395, 267, and 117 bp from E. multilocularis, Taenia spp., and E. granulosus were analyzed by $2 \%$ agarose gel electrophoresis, and the 380 and 300 bp fragments from $T$. canis and T. leonina were electrophoresed on $1.5 \%$ agarose gel stained with ethidium bromide, and visualized with a chemiluminescence imaging system (Syngene, Cambridge, UK).

\section{Sequencing}

For species identification of amplified 267 and 117 bp fragments, PCR-positive samples were subjected to single PCR using Cest 3 and Cest5 primer pairs for Taenia spp. and Cest 4 and Cest5 primer pairs for E. granulosus. PCR products were purified using the MinElute PCR purification Kit (Qiagen, Germany) according to the manufacturer's instructions and directly sequenced at the Bioneer Co. (Daejeon, South Korea) with the primers Cest 4 and Cest $5_{\text {seq }}$ for E. granulosus s.s. and Taenia spp., respectively.

\section{Statistical analyses}

The frequency of parasite occurrence in the samples and respective $95 \%$ confidence intervals (CI) were calculated using SPSS 18 software (SPSS Inc., Chicago, IL, USA).

\section{Results}

\section{Microscopic analysis}

Of the 167 fecal samples examined by light microscopy, 65 (38.9\%; 95\% CI: 32-46) samples were positive for ascarid and taeniid-like eggs. Of these, 43 (25.7\%; 95\% CI: 20-33) were identified as taeniid-like, 18 (10.8\%; 95\% CI: $7-16)$ as ascarid, and four (2.4\%; 95\% CI: 1-6) showed mixed infection with taeniid-like and ascarid eggs.

\section{PCR analysis}

The multiplex PCR revealed mono-infections by E. granulosus in seven (4.2\%; 95\% CI: 2-8) and Taenia spp. in 27 (17.4\%; 95\% CI: 11-23) while 13 (6.6\%; 95\% CI: 5-13) showed mixed infection by E. granulosus, Taenia spp., T. canis, and T. leonina. Echinococcus multilocularis infection was not identified in any sample (Fig. 1).

Of the 167 samples, 22 (13.2\%; 95\% CI: 9-19) were identified as ascarid by light microscopy. However, PCR results showed that 25 samples were infected with ascarid. Of these, T. canis was detected in nine (5.4\%; $95 \%$ CI: 3-10) and T. leonina in 16 (9.6\%; 95\% CI: 6-15) 


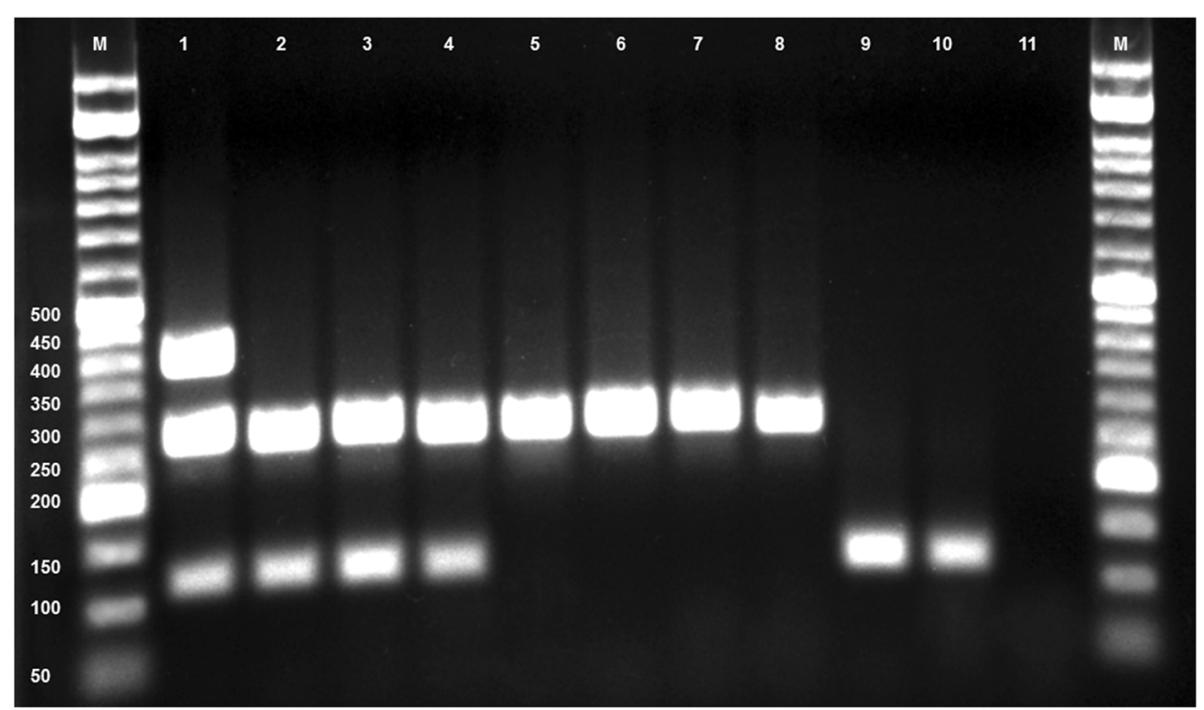

Fig. 1 Multiplex PCR amplification of mitochondrial genes of Echinococcus granulosus and Taenia spp. from eggs in feces of dogs from Khuzestan Province, Iran. Lane M, 50 bp DNA ladder; Lane 1, positive control, a mixture of standard DNA of E. multilocularis (395 bp), E. granulosus (117 bp), and T. hydatigena (267 bp); Lane 2-10, positive samples; Lane 11 negative control

samples. Two samples (1.2\%; 95\% CI: 0-4) showed co-infection by both species.

Based on PCR results, fifty $(29.9 \%)$ dogs showed mono-infection, and 15 (9\%) had mixed infections, including 12 (7.2\%; 95\% CI: 4-12) infected with two, and three $(1.8 \%$; 95\% CI: $1-5)$ infected with three, genera of the identified parasites. Taenia spp. were the most frequently detected parasites (24\%), with E. granulosus, found in 18 investigated dogs $(10.8 \%)$, the second most common (Table 1).

\section{DNA sequences analysis}

The obtained sequences were edited and aligned in MEGA 6.0 using ClustalW and compared to published GenBank sequences of E. granulosus s.s. (G1) (AF297617),

Table 1 Number and frequency (\%) of single and co-infections with Echinococcus granulosus, Taenia spp.., Toxocara canis, and Toxascaris leonina in fecal samples by PCR

\begin{tabular}{lll}
\hline Parasite species & N (\%) & $95 \% \mathrm{Cl}$ \\
\hline E. granulosus & $7(4.2 \%)$ & $2-8$ \\
Taenia spp. & $27(16.2 \%)$ & $11-23$ \\
T. canis & $5(3 \%)$ & $1-7$ \\
T. leonina & $11(6.6 \%)$ & $4-11$ \\
E. granulosus, Taenia spp. & $9(5.4 \%)$ & $3-10$ \\
Taenia spp., T. leonina & $1(0.6 \%)$ & $0-3$ \\
T. canis, T. leonina & $2(1.2 \%)$ & $0-4$ \\
E. granulosus, Taenia spp., T. canis & $1(0.6 \%)$ & $0-3$ \\
E. granulosus, Taenia spp., T. leonina & $1(0.6 \%)$ & $0-3$ \\
Taenia spp., T. leonina, T. canis & $1(0.6 \%)$ & $0-3$ \\
\hline
\end{tabular}

E. granulosus s.s. (G3) (KJ559023), Taenia hydatigena (GQ228819), Taenia multiceps (GQ228818), Taenia ovis (AB731675), and Taenia serialis (AB731674) by BLAST.

Ten isolates with sufficient DNA were sequenced. The obtained sequences of the rrnS gene of E. granulosus revealed the presence of E. granulosus s.s. (G1 genotype) in all sequenced samples.

The sequences from 30 Taenia spp. isolates revealed $T$. hydatigena in 18 (10.8\%), T. multiceps in five (3\%), T. serialis in three $(1.8 \%)$ dogs, and T. ovis in one $(0.6 \%)$ dog. The Cest $3 /$ Cest 5 primers are not specific for Taenia spp., thus they can detect non-taeniid cestodes such as Mesocestoides spp., Dipylidium caninum and Diphyllobothrium latum [32]. For this reason, the sequence of one sample identified Spirometra erinaceieuropaei voucher, and DNA sequencing of the remaining two amplicons was positive for Mesocestoides corti (Table 2). All isolates showed 99\% or greater identity with sequences in the GenBank database. Our sequences were submitted to GenBank with the accession numbers listed in Table 2.

\section{Discussion}

The obtained results provide the first molecular evidence of E. granulosus genotype G1 in dogs from the rural areas of Khuzestan Province, southwestern Iran. The previous studies showed that sheep and camel strains (G1 and G6) are the main cases of CE in Iran $[13,19,34]$, in which $G 1$ is the most common and the primary causative agent of the majority of canine and human infection [20,35]. Our data are in agreement with the earlier studies that demonstrate the dominant genotypes in canids are G1-G3 genotypes [16, 20,34]. 
Table 2 Number of infections and frequency (\%) of Echinococcus granulosus, Taenia spp., Spirometra erinaceieuropaei voucher, and Mesocestoides corti isolated from dog feces

\begin{tabular}{llll}
\hline Species & $\mathrm{N}(\%)$ & $95 \% \mathrm{Cl}$ & Accession numbers \\
\hline Taenia multiceps & $5(3)$ & $1-7$ & LC111533-LC111537 \\
Taenia serialis & $3(1.8)$ & $1-5$ & LC113990-LC113992 \\
Taenia ovis & $1(0.6)$ & $0-3$ & LC113989 \\
Echinococcus granulosus s.s. (G1) & $10(6)$ & $3-11$ & LC102348, LC114210-LC114215 \\
Taenia hydatigena & $18(10.8)$ & $7-16$ & LC114273-LC114275 \\
Spirometra erinaceieuropaei voucher & $1(0.6)$ & $0-3$ & LC106307-LC106308, LC106356-LC106360, LC107625-LC107626 \\
Mesocestoides corti & $2(1.2)$ & $0-4$ & - \\
\hline
\end{tabular}

The finding prevalence of $10.8 \%$ for E. granulosus are along with studies carried out in other parts of Iran that have shown a prevalence of $7.4 \%$ in the southeast [36], $13.2 \%$ in the west [37], $16.9 \%$ in the northeast [17], $20 \%$ in the northwest [38], and $64 \%$ in the central part of the country [39]. Eslami et al. reported that $20 \%$ of farm dogs was infected with E. granulosus in the only study conducted in the study area. It is possible that the lower prevalence in this survey compared to the previous study is due to factors such as the studied population. In the study conducted by Eslami et al., only farm dogs that spent more time roaming were investigated; therefore, they always had easy access to discarded offal, which resulted in a higher chance of infection [24]. In addition, it should be considered that the rate of infection has decreased during the recent years in all over the country in human and dogs. This phenomenon may be results of a growing awareness of people and improving the quality of healthcare in Iran. High reported prevalence of infection in the northwest [38], and central part of the country [39] might be related to geographical locations and environmental temperature. Khuzestan Province has dry and hot weather, and in summer the temperature can rise to over $50{ }^{\circ} \mathrm{C}$ in some regions, including Ahvaz [40], so it may decrease the survival of eggs in summer. Under optimal conditions such as high precipitation, moist soil, and low temperature, taeniid and ascarid eggs can survive at least for one year, which provide opportunities for human infections [41, 42]. Despite the fact that climatic conditions in Khuzestan Province in summer may decrease the possibility of the infection, the role of domestic dogs in environmental contamination and human infection should not be ignored.

Rural inhabitants comprise approximately $15 \%$ of the over 4.5 million people living in Khuzestan Province [27]. In many rural homes, large populations of dogs are kept for being shepherd and guard dogs, so the E. granulosus-infected dogs play key roles in the transmission of echinococcosis to humans through contamination of soil with its feces as mentioned in previous studies [14, 24].
In recent years, because of El Niño Southern Oscillation (ENSO), dust storms have affected Iran and other Persian Gulf countries. Based on the reported data, one of the main targets of dust storms is the southwest of Iran [43]. Given that the streets of Khuzestan villages are muddy and unpaved, these can lead to the spread of parasitic agents and the contamination of human environment, food, vegetables, and, particularly, water supplies of rural inhabitants. Furthermore, it is a serious threat to other provinces near to Khuzestan.

Despite a report of human AE from Khuzestan Province [23], its causative agent, E. multilocularis, was not identified in the dogs examined. Therefore, it seems that the reported case [23] should be infected in a place other than Khuzestan with more suitable environment conditions for $E$. multilocularis life cycle. Although, with respect to the lower prevalence of $E$. multilocularis infection among domestic dogs compared to other definitive hosts such as red fox $[17,44]$, for reaching to the stringent deduction a widespread investigation covering more samples and areas is essential.

The occurrence of $T$. hydatigena, $T$. multiceps, and $T$. ovis in investigated dogs indicates the potential of domestic dogs as definitive hosts that may acquire infection by scavenging on infected domestic herbivorous mammals, principally sheep carcasses. Lethal infection with some Taenia spp., particularly $T$. multiceps, in intermediate hosts such as sheep, is significant, causing considerable economic losses $[45,46]$. These species have also been reported to have zoonotic importance, with several human cases recorded worldwide [47]. Taenia hydatigena (53\%) and T. multiceps (3-40\%) are among the most prevalent Taenia spp. in dogs in Iran $[45,48]$, with the economic losses due to T. multiceps infection in sheep estimated at 15,700 USD in 1990-1992 [49]. Despite economic importance caused by T. hydatigena and T. multiceps in intermediate hosts, no molecular epidemiological data were recorded in definitive hosts from southwestern Iran. Furthermore, molecular examinations confirmed the presence of S. erinaceieuropaei voucher 
infection in one dog. According to our knowledge, this is the first report of S. erinaceieuropaei voucher in Iranian dogs. Spirometra erinaceieuropaei, is one of the most important cestodes, causes sparganosis in human [50]. Human infection occurs mainly through consumption of raw or undercooked meat of infected animals with plerocercoids, such as snakes and frogs, water contaminated with cyclops, or by using frog or snake flesh in traditional medical practices [51]. Moreover, the existence of $M$. corti (syn. $M$. vogae) in two dogs confirmed by sequencing results. This tapeworm is mainly a mice parasite but is also a human life-threatening cestodes similar to $E$. multilucularis and Taenia solium [52]. Although, another species of this genus, $M$. lineatus, has been reported from stray dogs $(26.5 \%)$ in the west of Iran [37], this is the first time that M. corti reported in dogs of Iran.

Other important zoonotic helminthes, T. canis (5.4\%) and T. leonina (9.6\%), was reported in the present study. There is a dramatically difference in the reported prevalence of $T$. canis infection in domestic and stray dogs from $60 \%$ in the north [39] and $22 \%$ in the central [6] to $6 \%$ in the western [37], $4.3 \%$ in southeast [53] and 5.4\% in southwestern (this study) of Iran. It seems that the observed differences in the investigated areas is dependent on different factors such as the geographical location, temperature and humidity, soil type, vegetation cover, or the age of the examined dogs $[41,54]$.

\section{Conclusions}

This study has demonstrated relatively high prevalence of zoonotic parasites (38.9\%) and the first reports of $S$. erinaceieuropaei and $M$. corti in domestic dogs from Khuzestan Province. Despite the lower prevalence of $E$. granulosus and T. canis compare to other parts of the country, the large population of dogs, particularly stray dogs are significant. Therefore, these findings are important in two respects: the first, the obtained data indicate a serious risk for echinococcosis and toxocarosis, the latter, these findings reflect a possible role of dogs in transmission of human sparganosis in the area. Despite the fact that in Khuzestan Province people avoid traditional medical practices by using snake or frog flesh, the risk of transmission by contaminated water should not be ignored. Thus, more investigations on S. erinaceieuropaei and $M$. corti among Khuzestan rural dogs is essential. Moreover, control and preventive programs, including health education, anthelminthic treatment of dogs, and supervision of home slaughtering, should be implemented to reduce the transmission of zoonotic parasites to the inhabitants of villages.

\section{Abbreviations}

${ }^{\circ} \mathrm{C}$ : Centigrade; AE: Alveolar echinococcosis; BLAST: Basic Local Alignment Search Tool; bp: Base pair; CE: Cystic echinococcosis; Cest: Cestodes; Cl: Confidence Interval; DNA: Deoxyribonucleic acid; E: East; E. granulosus: Echinococcus granulosus; E. multilocularis: Echinococcus multilocularis; Echinococcus granulosus s.s.: Echinococcus granulosus sensu stricto; ENSO: El Niño Southern Oscillation; G: Genotype; g: Gram; g: Gravity; h: Hour; ITS: Internal transcribed spacer; $\mathrm{km}^{2}$ : Square kilometre; $M$. corti: Mesocestoides corti; M. lineatus: Mesocestoides lineatus; min: Minute; ml: Milliliter; mm: Millimeter; N: North; nad1: NADH dehydrogenase subunit 1; PCR: Polymerase chain reaction; rrnS: Small subunit of ribosomal RNA; S. erinaceieuropaei voucher: Spirometra erinaceieuropaei voucher; sec: Second; seq: Sequencing; spp.: The plural form of species; SPSS: Statistical Package for the Social Sciences; T. canis: Toxocara canis; T. hydatigena: Taenia hydatigena; T. leonina: Toxascaris leonina; T. multiceps: Taenia multiceps; T. ovis: Taenia ovis; T. serialis: Taenia serialis; Tcan: T canis; Tleo: T leonina; UK: United Kingdom; USD: United States Dollar; $\mu$ l: Microliter; $\mu \mathrm{m}$ : Micrometer; $\mu$ M: Micromolar

\section{Acknowledgments}

The authors gratefully acknowledge the inhabitants of villages and the dog owners for their help in sample collection.

\section{Funding}

This project was supported by Infectious and Tropical Diseases Research Center, Health Research Institute, Ahvaz Jundishapur University of Medical Sciences, Ahvaz, Iran grant P/3065.

\section{Availability of data and materials}

The datasets used and/or analysed during the current study available from the corresponding author on reasonable request.

\section{Authors' contributions}

MB conceived and designed the study. MB carried out laboratory analyses. All the authors contributed to the data analysis, interpretation and preparation of the manuscript. The manuscript was approved by all the authors.

\section{Ethics approval and consent to participate}

Prior to collection of fecal samples, the required information regarding the study was provided to dog owners and the permission for sample collection was obtained. The protocol of this investigation was reviewed and approved by the Ethics Committee of Ahvaz Jundishapur University of Medical Sciences (Approval No OG-91137).

Consent for publication

Not applicable.

\section{Competing interests}

The authors declare that they have no competing interests.

\section{Publisher's Note}

Springer Nature remains neutral with regard to jurisdictional claims in published maps and institutional affiliations.

\section{Author details}

'Infectious and Tropical Diseases Research Center, Health Research Institute, Ahvaz Jundishapur University of Medical Sciences, Ahvaz, Iran. ${ }^{2}$ Department of Parasitology, School of Medicine, Ahvaz Jundishapur University of Medical Sciences, Ahvaz, Iran. ${ }^{3}$ Department of Parasitology and Mycology, School of Medicine, Iran University of Medical Sciences, Tehran, Iran. ${ }^{4}$ Institute of Health Research, Thalassemia and Hemoglobinopathy Research Center, Ahvaz Jundishapur University of Medical Sciences, Ahvaz, Iran.

Received: 13 April 2017 Accepted: 19 June 2018

Published online: 25 July 2018

\section{References}

1. Deplazes P, van Knapen F, Schweiger A, Overgaauw PA. Role of pet dogs and cats in the transmission of helminthic zoonoses in Europe, with a focus on echinococcosis and toxocarosis. Vet Parasitol. 2011;182(1):41-53.

2. Soriano SV, Pierangeli NB, Roccia I, Bergagna HF, Lazzarini LE, Celescinco A, et al. A wide diversity of zoonotic intestinal parasites infects urban and rural dogs in Neuquen, Patagonia, Argentina. Vet Parasitol. 2010;167(1):81-5.

3. Lee RM, Moore LB, Bottazzi ME, Hotez PJ. Toxocariasis in North America: a systematic review. PLoS Negl Trop Dis. 2014;8(8):e3116. 
4. Blaszkowska J, Wojcik A, Kurnatowski P, Szwabe K. Geohelminth egg contamination of children's play areas in the city of Lodz (Poland). Vet Parasitol. 2013;192(1-3):228-33.

5. Borecka A. Prevalence of intestinal nematodes of dogs in the Warsaw area. Poland Helminthologia. 2005;42(1):35-9.

6. Daryani A, Sharif M, Amouei A, Gholami S. Prevalence of Toxocara canis in stray dogs, northern Iran. Pak J Biol Sci. 2009;12(14):1031-5.

7. Awoke $\mathrm{E}$, Bogale $\mathrm{B}$, Chanie M. Intestinal nematode parasites of dogs: prevalence and associated risk factors. Int J Anim Vet Adv. 2011;3(5):374-8.

8. Strube C, Heuer L, Janecek E. Toxocara spp. infections in paratenic hosts. Vet Parasitol. 2013;193(4):375-89.

9. Eckert J, Deplazes P. Biological, epidemiological, and clinical aspects of echinococcosis, a zoonosis of increasing concern. Clin Microbiol Rev. 2004; 17(1):107-35.

10. Carmena D, Cardona GA. Echinococcosis in wild carnivorous species: epidemiology, genotypic diversity, and implications for veterinary public health. Vet Parasitol. 2014;202(3-4):69-94.

11. Romig T, Ebi D, Wassermann M. Taxonomy and molecular epidemiology of Echinococcus granulosus sensu lato. Vet Parasitol. 2015;213(3-4):76-84

12. McManus DP. Current status of the genetics and molecular taxonomy of Echinococcus species. Parasitology. 2013;140(13):1617-23.

13. Jenkins DJ, Romig T, Thompson RCA. Emergence/re-emergence of Echinococcus spp. a global update. Int J Parasitol. 2005:35(11-12):1205-19.

14. Zhang W, Zhang Z, Wu W, Shi B, Li J, Zhou X, et al. Epidemiology and control of echinococcosis in Central Asia, with particular reference to the People's Republic of China. Acta Trop. 2015;141, Part B:235-43.

15. Amer S, Helal IB, Kamau E, Feng Y, Xiao L. Molecular characterization of Echinococcus granulosus Sensu Lato from farm animals in Egypt. PLoS One. 2015;10(3):e0118509.

16. Rokni MB. Echinococcosis/hydatidosis in Iran. Iran J Parasitol. 2009;4(2):1-16.

17. Beiromvand M, Akhlaghi L, Fattahi Massom SH, Mobedi I, Meamar AR, Oormazdi $\mathrm{H}$, et al. Detection of Echinococcus multilocularis in carnivores in Razavi Khorasan province, Iran using mitochondrial DNA. PLoS Negl Trop Dis. 2011;5(11):e1379.

18. Fasihi Harandi M, Budke CM, Rostami S. The monetary burden of cystic echinococcosis in Iran. PLoS Negl Trop Dis. 2012;6(11):e1915.

19. Harandi MF, Hobbs RP, Adams PJ, Mobedi I, Morgan-Ryan UM, Thompson RC. Molecular and morphological characterization of Echinococcus granulosus of human and animal origin in Iran. Parasitology. 2002;125(4): 367-73.

20. Carmena D, Cardona GA. Canine echinococcosis: global epidemiology and genotypic diversity. Acta Trop. 2013;128(3):441-60.

21. Mobedi I, Bray RA, Arfaa F, Movafag K. A study on the Cestodes of carnivores in the northwest of Iran. J Helminthol. 1973:47(03):277-81.

22. Mobedi I, Sadighian A. Echinococcus multilocularis Leuckart, 1863, in red foxes, Vulpes vulpes Linn., in Moghan, Azerbaijan Province, northwest of Iran. J Parasitol. 1971:57(3):493.

23. Geramizadeh B, Nikeghbalian S, Malekhosseini SA. Alveolar echinococcosis of the liver: report of three cases from different geographic areas of Iran. Hepat Mon. 2012;12(9):e6143.

24. Eslami A, Hosseini SH. Echinococcus granulosus infection of farm dogs of Iran. Parasitol Res. 1998;84(3):205-7.

25. Ranjbar-Bahadori S, Lotfollahzadeh S, Vaezi G, Eslami A. Epidemiological study of the human cystic echinococcosis in Iran. Res J Parasitol. 2008;3:130-6.

26. Zarasvandi A, Carranza EJM, Moore F, Rastmanesh F. Spatio-temporal occurrences and mineralogical-geochemical characteristics of airborne dusts in Khuzestan Province (southwestern Iran). J Geochem Explor. 2011:111(3):138-51.

27. Census. Census of the Islamic Republic of Iran, Statistical Centre of Iran. https://www.amar.org.ir/. 2011.

28. Eckert J, Gottstein B, Heath D, Liu F-J. Prevention of echinococcosis in humans and safety precautions. J. Eckert, M.A. Gemmell, F.X. Meslin, Z.S. Pawlowski (Eds.), Manual on Echinococcosis in Humans and Animals: a Public Health Problem of Global Concern, OIE and WHO, Paris, France. 2001;238-248.

29. Mathis A, Deplazes P, Eckert J. An improved test system for PCR-based specific detection of Echinococcus multilocularis eggs. J Helminthol. 1996;70(03):219-22.

30. Verweij JJ, Pit DSS, Van Lieshout L, Baeta SM, Dery GD, Gasser RB, et al. Determining the prevalence of Oesophagostomum bifurcum and Necator americanus infections using specific PCR amplification of DNA from faecal samples. Tropical Med Int Health. 2001;6(9):726-31.
31. Borecka A, Gawor J. Modification of gDNA extraction from soil for PCR designed for the routine examination of soil samples contaminated with Toxocara spp eggs. J Helminthol. 2008;82(02):119-22.

32. Trachsel $D$, Deplazes $P$, Mathis $A$. Identification of taeniid eggs in the faeces from carnivores based on multiplex PCR using targets in mitochondrial DNA. Parasitology. 2007;134(06):911-20.

33. Jacobs DE, Zhu X, Gasser RB, Chilton NB. PCR-based methods for identification of potentially zoonotic ascaridoid parasites of the dog, fox and cat. Acta Trop. 1997;68(2):191-200.

34. Parsa F, Fasihi Harandi M, Rostami S, Sharbatkhori M. Genotyping Echinococcus granulosus from dogs from western Iran. Exp Parasitol. 2012;132(2):308-12.

35. Alvarez Rojas CA, Romig T, Lightowlers MW. Echinococcus granulosus sensu lato genotypes infecting humans - review of current knowledge. Int J Parasitol. 2014;44(1):9-18

36. Sharifi I, Zia-Ali N. The present status and intensity of Echinococcus granulosus infection in 391 stray dogs in rural and urban areas of the city of Kerman Iran. Iran J Public Health. 1996;25(3-4):13-20.

37. Dalimi A, Sattari A. Motamedi G. A study on intestinal helminthes of dogs, foxes and jackals in the western part of Iran. Vet Parasitol. 2006; 142(1-2):129-33.

38. Shariatzadeh S, Spotin A, Gholami S, Fallah E, Hazratian T, Mahami-Oskouei M, et al. The first morphometric and phylogenetic perspective on molecular epidemiology of Echinococcus granulosus sensu lato in stray dogs in a hyperendemic Middle East focus northwestern Iran. Parasit Vectors. 2015;8(1):1-10.

39. Eslami A, Ranjbar-Bahadori S, Meshgi B, Dehghan M, Bokaie S. Helminth infections of stray dogs from garmsar, Semnan province central iran. Iran J Parasitol. 2010;5(4):37-41.

40. Mombeni HA, Rezaei S, Nadarajah S, Emami M. Estimation of water demand in Iran based on SARIMA models. Environmental Modeling \& Assessment. 2013;18(5):559-65.

41. Macpherson CNL. The epidemiology and public health importance of toxocariasis: a zoonosis of global importance. Int J Parasitol. 2013;43(12-13):999-1008.

42. Burlet $P$, Deplazes $P$, Hegglin D. Age, season and spatio-temporal factors affecting the prevalence of Echinococcus multilocularis and Taenia taeniaeformis in Arvicola terrestris. Parasit Vectors. 2011:4:6.

43. Nabavi SO, Haimberger L, Samimi C. Climatology of dust distribution over West Asia from homogenized remote sensing data. Aeolian Res. 2016;21:93-107.

44. Dyachenko V, Pantchev N, Gawlowska S, Vrhovec MG, Bauer C. Echinococcus multilocularis infections in domestic dogs and cats from Germany and other European countries. Vet Parasitol. 2008;157(3-4):244-53.

45. Rostami S, Beech RN, Salavati R, Baneshi MR, Kamyabi H, Harandi MF. Morphometric analysis of larval Rostellar hooks in Taenia multiceps of sheep in Iran and its association with mitochondrial gene variability. Iran J Parasitol. 2013;8(4):579-85.

46. Varcasia A, Tamponi C, Tosciri G, Pipia AP, Dore F, Schuster RK, et al. Is the red fox (Vulpes vulpes) a competent definitive host for Taenia multiceps? Parasit Vectors. 2015:8:491

47. Rostami S, Salavati R, Beech RN, Sharbatkhori M, Babaei Z, Saedi S, et al. Cytochrome c oxidase subunit 1 and 125 ribosomal RNA characterization of Coenurus cerebralis from sheep in Iran. Vet Parasitol. 2013;197(1-2):141-51.

48. Rostami S, Salavati R, Beech RN, Babaei Z, Sharbatkhori M, Baneshi MR, et al. Molecular and morphological characterization of the tapeworm Taenia hydatigena (Pallas, 1766) in sheep from Iran. J Helminthol. 2015;89(02):150-7.

49. Oryan A, Moghaddar N, Gaur SNS. Metacestodes of sheep with special reference to their epidemiological status, pathogenesis and economic implications in Fars Province. Iran Vet Parasitol. 1994;51(3-4):231-40.

50. Zhang X, Cui J, Liu LN, Jiang P, Wang H, Qi X, et al. Genetic structure analysis of Spirometra erinaceieuropaei isolates from central and southern China. PLoS One. 2015;10(3):e0119295.

51. Liu Q, Li M-W, Wang Z-D, Zhao G-H, Zhu X-Q. Human sparganosis, a neglected food borne zoonosis. Lancet Infect Dis. 2015;15(10):1226-35.

52. Vendelova E, Hrčková G, Lutz MB, Brehm K, Nono JK. In vitro culture of Mesocestoides corti metacestodes and isolation of immunomodulatory excretory-secretory products. Parasite Immunol. 2016;38(7):403-13.

53. Mirzaei M, Fooladi M. Prevalence of intestinal helminthes in owned dogs in Kerman city, Iran. Asian Pac J Trop Med. 2012;5(9):735-7.

54. Okulewicz A, Perec-Matysiak A, Buńkowska K, Hildebrand J. Toxocara canis, Toxocara cati and Toxascaris leonina in wild and domestic carnivores. Helminthologia. 2012:49(1):3-10. 